\title{
Biomasa aérea y modelos alométricos para Acacia pennatula, en condiciones naturales del trópico seco Nicaragüense
}

\author{
Kenny López Benavides ${ }^{1}$ \\ Idania del Rosario Castillo Castillo² \\ Doris del Carmen Altamirano Espino
}

\section{RESUMEN}

La investigación se realizó en los reductos forestales de vegetación secundaria ubicados en la Estación Experimental - El Limón, Estelí, Nicaragua. Con el objetivo de evaluar la producción de biomasa forrajera y leña de Carbón (Acacia pennatula Schltdl y Cham) Benth, en condiciones naturales de vegetación secundaria en el trópico seco Nicaragüense. Con este fin, el experimento se enfoco en dos fases: 1) determinar la producción de biomasa forrajera y leña, 2) generar ecuaciones alométricas para estimar la producción de biomasa forrajera y leña.

Se seleccionaron 26 individuos de Carbón, comprendidos en un rango amplio de altura y diámetro a la altura de pecho. Posteriormente se efectúo una poda de homogenización a una altura de 1.8 metros, a partir del suelo, con la finalidad de que cada individuo mostrará su capacidad productora de biomasa, durante un período de 6 meses. Después de éste periodo de acumulación se cosecho la biomasa, la cual se dividió en dos fracciones: forraje y leñosa. Para cada individuo se midió el diámetro a la altura del pecho, el número de rebrotes, diámetro de la base, la longitud de cada rebrote y el peso fresco de forraje y leña.

Se tomaron muestras frescas de cada fracción por individuo, las cuales se secaron a $60{ }^{\circ} \mathrm{C}$ por 48 horas, hasta alcanzar un peso constante. Posteriormente, se estimó la producción de biomasa seca total de ambas fracciones por árbol, a través del modelo de predicción lineal " $\mathrm{y}$ " $=0.4357 \mathrm{x}$ 8.3423, altamente significativo $(\mathrm{p}=0.0001 \mathrm{y} \mathrm{R}=0.988)$.

Según los coeficiente de correlación de Pearson, las variables que asociaron a la producción de forraje fueron: diámetro de la base y longitud del rebrote $(\mathrm{R} 2=0.635)$, número de rebrotes $(\mathrm{R} 2=$ $0.647)$ y diámetro a la altura de pecho $(\mathrm{R} 2=0.722)$. Mientras que la leña está asociada al diámetro de la base y longitud del rebrote $(\mathrm{R} 2=0.617$ y 0.779$)$, respectivamente. Todas las correlaciones antes mencionadas resultaron estadísticamente significativas $(\mathrm{p}<0.05)$. Las variables evaluadas se ajustaron a modelos de regresión lineal.

Palabras clave: Biomasa, forraje, leña y ecuaciones alométricas

1 UNAN-Managua/FAREM-Estelí. Correo electrónico: kenny.lb@hotmail.com

2 UNAN-Managua/FAREM-Estelí. Correo electrónico: idacastillo17@yahoo.com 


\title{
Aboveground biomass and allometric models for Acacia pennatula in natural dry tropical conditions Nicaraguan
}

\author{
Kenny López Benavides ${ }^{1}$ \\ Idania del Rosario Castillo Castillo² \\ Doris del Carmen Altamirano Espino
}

\begin{abstract}
The research was conducted in the receptacles of secondary growth forest located at the Experimental Station - El Limon, Estelí, Nicaragua; in order to evaluate the production of forage biomass and firewood Coal (Acacia pennatula Schltdl and Cham) Benth, under natural conditions of secondary vegetation in the Nicaraguan dry tropics. To this end, the experiment focused on two phases: 1) determine the production of forage biomass and firewood, 2) generate allometric equations to estimate biomass production of fodder and firewood.
\end{abstract}

Twenty six individuals of coal included in a wide range of height and diameter at breast height were selected. Later, homogenization pruning was performed at a height of 1.8 meters above the ground, in order that each individual could show its capacity to produce biomass for a period of 6 months. After this period of accumulation, biomass was harvested which was divided into two fractions: forage and woody. For each individual diameter was measured at breast height, the number of regrows, base diameter, the length of each regrowth and fresh weight of fodder and firewood.

Fresh samples of each fraction were taken per individual, which were dried at $60^{\circ} \mathrm{C}$ for 48 hours until reaching a constant weight. Subsequently, the production of both fractions of the total dry biomass per tree was estimated, through the linear prediction model "and" $=0.4357 \mathrm{x}-8.3423$, highly significant $(\mathrm{p}=0.0001$ and $\mathrm{R}=0.988)$.

According to the Pearson correlation coefficient, the variables associated with forage production were: base diameter and length of the regrowth $(\mathrm{R} 2=0.635)$, number of regrows $(\mathrm{R} 2=0.647)$ and diameter at breast height $(\mathrm{R} 2=0.722)$. While wood is associated with the base diameter and length of the regrowth $(\mathrm{R} 2=0.617$ and 0.779$)$, respectively. All of the above correlations were statistically significant $(\mathrm{p}<0.05)$. The evaluated variables were fitted to linear regression models.

Keywords: Biomass, fodder, firewood and allometric equations

1 UNAN-Managua/FAREM-Estelí. E-mail: kenny.lb@hotmail.com

2 UNAN-Managua/FAREM-Estelí. E-mail: idacastillo17@yahoo.com 


\section{INTRODUCCIÓN}

En las regiones tropicales los sistemas de alimentación de rumiantes están basados en la utilización de pastos, los cuales presentan limitaciones de calidad y cantidad en la época seca que puede extenderse durante periodos de hasta 6 meses consecutivos con escasez de lluvias (Pérez et al, 2008). No obstante, las zonas tropicales poseen una gran diversidad de especies vegetales, las cuales tienen un alto valor biológico debido a las características nutritivas y a los diversos beneficios que aportan al ambiente.

El pastoreo del ganado en gramíneas naturales y mejoradas, así como el uso de los recursos proteicos forrajeros provenientes de la contribución de los árboles y los arbustos, es tan antiguo como su propia existencia. Sin embargo, los sistemas modernos de producción ganadera derivaron hacia el uso de tecnologías intensivas, basadas en otros recursos energético-proteicos que pudieran complementar las dietas a base de pastos, o el empleo de sistemas de corte y acarreo de forraje en grandes áreas de monocultivo (Simón et al, 2011).

En algunos países de Centroamérica como Costa Rica, Nicaragua y El Salvador se ha producido una grave reducción de la cobertura arbórea. La ganadería basada en pastos nativos ha sido la causante del mayor cambio en los paisajes rurales hasta llegar a una escala continental y debe reconocerse como un proceso de enormes repercusiones ambientales y sociales (Bennett y Hoffmann 1992, Steinfeld 2000 citado por Pérez, 2006).

Múltiples investigaciones se han realizado en ésta temática, López et al (2003), determinaron relaciones alométricas para predecir biomasa forrajera y leña en Acacia pennatula y Guazuma ulmifolia, en condiciones de vegetación secundaria, en dos comunidades del norte de Chiapas, México. De igual manera, Guillén et al (2007), establecieron ecuaciones de predicción para la producción de forraje de Cercidium floridum, en baja California sur, México. También, Etchevers et al (2002), estimaron la biomasa aérea mediante el uso de relaciones alométricas en seis especies arbóreas en Oaxaca, México. Nieto 2000, determino la contribución de Acacia pennatula a la productividad agroforestal sostenible de la Reserva Natural MiraflorMoropotente, Estelí, Nicaragua.

Nicaragua tiene una riqueza considerable en árboles forrajeros, que actualmente no tienen aprovechamiento óptimo. Al mismo tiempo, hay gran interés y apreciación de la importancia de estos árboles por parte de los productores. Por tal razón existe la oportunidad de fomentar su siembra y aprovechamiento, con bastante posibilidad de éxito (Durr, 1992).

En vista de lo anterior, surge la necesidad de evaluar la producción de biomasa forrajera y leña de Carbón, en condiciones de vegetación secundaria. Para lo cual se hizo necesario: determinar la producción de biomasa aérea, el modelo de regresión de mejor ajuste y generar ecuaciones alométricas para estimar biomasa forrajera. A fin de continuar promoviendo el uso del Carbón, como una especie de interés multipropósito para el diseño de sistemas silvopastoriles y mejorar la producción de bienes y servicios ecosistémicos en las fincas, como medidas de adaptación y mitigación ante el cambio global.

\section{MATERIALES Y MÉTODOS}

\section{Área de estudio}

La investigación se realizó durante el año 2012, en los parches forestales de la Estación Experimental "El Limón" para el estudio del trópico seco, adscrita a la Universidad Nacional Autónoma de Nicaragua (UNAN-Managua/FAREM-Estelí). Ubicada en las coordenadas UTM 0568720 y 1443707 en una cota altitudinal de 890 m.s.n.m. Con rangos mensuales de temperatura que fluctúan entre 16 y $33^{\circ} \mathrm{C}$ y una 
precipitación media anual de $804 \mathrm{~mm}$. El suelo, es franco - arcilloso de color café amarillento con abundantes rocas blandas (Peguero et al, 2011).

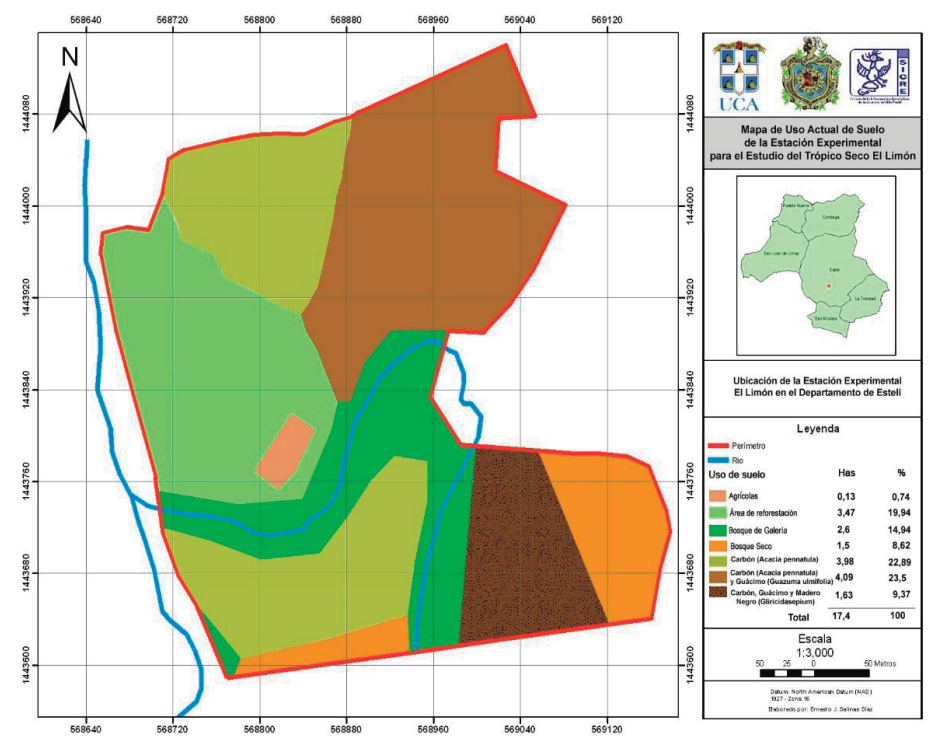

Figura 1: Estación Experimental “El Limón”.

\section{Cuantificación de biomasa aérea (forraje y leña)}

Se seleccionaron 26 individuos $(\mathrm{n}=26)$ de Carbón (Acacia pennatula), comprendidos en un rango amplio de altura y Diámetro a la Altura de Pecho $($ DAP $=1.3$ metros a partir del suelo), basándose en la metodología de Salazar (1989), que recomienda la utilización de 16 á 36 individuos para la cuantificación de forraje arbóreo.

Posteriormente se efectúo una poda de homogenización a una altura de 1.8 metros, a partir del suelo, con la finalidad de que cada individuo mostrara su capacidad productora de biomasa (forraje y leña), durante un período de 6 meses (Salazar, 1989). Después de éste periodo de acumulación se cosecho la biomasa, la cual se dividió en dos fracciones:

1. una fracción de biomasa forrajera (hojas y tallos tiernos, con diámetros menores o iguales á $0.5 \mathrm{~cm}$ ),

2. una fracción de biomasa leñosa (tallos, con diámetros mayores á $0.5 \mathrm{~cm})$. Ambas fracciones fueron pesadas por separado para determinar la producción de las mismas.

Para cada individuo se midió: el diámetro a la altura del pecho, el número de rebrotes, diámetro de la base, la longitud de cada rebrote y el peso fresco de forraje y leña.

Se tomaron muestras frescas de ambas fracciones, de $300(\mathrm{~g})$ por individuo, las cuales se secaron a 60 ${ }^{\circ} \mathrm{C}$ por 48 horas, hasta alcanzar un peso constante. Se utilizó en una estufa digital con capacidad de 150 de litros y temperatura máxima de $200^{\circ} \mathrm{C}$. Los pesos secos obtenidos, permitieron estimar la producción de biomasa aérea seca total por árbol, a través de los modelos de regresión lineal simple generados para forraje y leña: " $\mathrm{y}$ " $=0.4357 \mathrm{x}-8.3423,(\mathrm{R}=0.988, \mathrm{p}$ $=0.0001) \mathrm{y}$ “ $\mathrm{y}$ " $=0.4321 \mathrm{x}-3.3381,(\mathrm{R}=0.978, \mathrm{p}=$ $0.0001)$, respectivamente.

\section{Análisis estadístico}

Se determinó la normalidad de los datos, a través de una prueba de Kolmogorov-Smirnov $(\mathrm{K}-\mathrm{S})$, para una muestra. Posteriormente se realizó estadística paramétrica a través de correlaciones y modelos de mejor ajuste a través de regresiones simples, tomando como referencia los coeficientes de correlación de Perason, (R) y determinación (R2), respectivamente. Además se realizaron transformaciones de modelos potenciales $(y=a * x b)$ a lineales $(y=b * x+a)$, obteniendo los logaritmos naturales de las variables estudiadas.

El software utilizado para el procesamiento de la información fue infoStat versión 2013. 


\section{RESULTADOS Y DISCUSIÓN}

\section{Producción de biomasa forrajera y leña de Carbón} (Acacia pennatula).

La producción mínima y máxima de biomasa seca forrajera en Carbón fue de 0.4 y 731.8 (g), respectivamente. Mientras que la producción promedio de biomasa seca forrajera fue de $166.5 \pm 48.33(\mathrm{~g})$, en un período de seis meses de acumulación.

El mayor porcentaje de producción de biomasa seca total, durante el periodo de acumulación la obtuvo la biomasa comestible en relación a la leñosa (Tabla 1). Este resultado es similar con lo propuesto por Stür et al (1994), que el momento indicado para cosechar el follaje de las especies arbóreas es cuando existe la máxima productividad de material comestible; esto ocurre cuando dicha fracción representa del 50 al 60\% del total producido.

Tabla 1: Producción de biomasa por fracción.

\begin{tabular}{ccc}
\hline Fracción de biomasa & Producción $(\mathbf{g})$ & Porcentaje \\
\hline Forraje & 2834 & 63 \\
Leña & 1687 & 37 \\
Total & 4520 & 100 \\
\hline
\end{tabular}

Según el coeficiente de correlación de Pearson, demuestra un fuerte grado de asociación lineal positiva entre las variables que mejor determinaron la producción total por individuo de biomasa forrajera seca: diámetro de la base del rebrote, longitud del rebrote, número de rebrotes, diámetro a la altura de pecho. Las correlaciones entre producción total por individuo de biomasa seca forrajera y las variables antes mencionadas resultaron estadísticamente significativas $(p<0.05)$ y valores de correlación cercanos a 1 . No obstante la biomasa forrajera seca total por individuo no está asociada con la altura de los árboles, por presentar un valor de correlación próximo a cero. A demás la correlación resulto no significativa $(\mathrm{p}>0.05)$, (Tabla 2). Este resultado es similar a lo encontrado por
Nieto (2000), quien encontró los valores más bajos de correlación entre la altura y producción de forraje en A. pennatula. Considerando que es de esperarse ya que el carbón no pasa los $12 \mathrm{~m}$ de altura, los que se alcanzan rápidamente.

Tabla 2: Correlación de la producción de biomasa forrajera seca total

\begin{tabular}{cccccc}
\hline $\begin{array}{c}\text { Correlación de } \\
\text { Pearson }\end{array}$ & $\begin{array}{c}\text { Diámetro de } \\
\text { la base del } \\
\text { rebrote }(\mathbf{c m})\end{array}$ & $\begin{array}{c}\text { Longitud } \\
\text { del rebrote } \\
(\mathbf{c m})\end{array}$ & $\begin{array}{c}\text { Número } \\
\text { de rebotes }\end{array}$ & $\begin{array}{c}\text { Altura } \\
(\mathbf{m})\end{array}$ & Dap cm \\
\hline $\begin{array}{c}\text { Biomasa forrajera } \\
\text { seca total }(\mathrm{g})\end{array}$ & $0.635(* *)$ & $0.635(* *)$ & $0.647(* *)$ & -0.041 & $0.722(* *)$ \\
Sig. bilateral & 0.006 & 0.006 & 0.005 & 0.877 & 0.001 \\
$\mathrm{n}$ & 17 & 17 & 17 & 17 & 17 \\
\hline
\end{tabular}

** La correlación es significativa a nivel 0.01 (bilateral)

Las variables relacionadas a la producción de biomasa forrajera seca, evidencian un comportamiento potencial de la forma $y=a * x b$. Transformadas a modelos lineales de tipo $y=b * x+a$, donde " $a$ " $y$ " $b$ " son estimadores de parámetros, "y" es la biomasa forrajera seca (g), "x" representa a las cuatro variables alométricas estudiadas (diámetro de la base del rebrote, longitud del rebrote, número de rebrotes y diámetro a la altura de pecho). Estas fueron transformadas obteniendo sus logaritmos naturales, expresados matemáticamente como: $\left.\ln a+b^{*} \ln x\right)$, (figuras 1, 2, 3 y 4). Al respecto Piñol y Martínez (2006) mencionan que muchas variables de interés ecológico presentan mayoritariamente relaciones de tipo potencial. Así, por ejemplo la masa total de los árboles guarda una estrecha relación con el diámetro del tronco.

Además demuestran una relación lineal positiva y directamente proporcional entre la biomasa seca forrajera (variable dependiente), en función de las variables evaluadas (variables independientes). Lo cual indica, la cantidad en que la recta se eleva (pendiente), por cada unidad de incremento en las variables independientes. 
Los modelos alométricos de tipo lineal para las variables: diámetro de la base del rebrote, longitud del rebrote, número de rebrotes y diámetro a la altura de pecho (Dap), describen coeficientes de determinación (R2), de 0.442, 0.691, 0.704 y 0.621, respectivamente Estos explican en valores porcentuales que la variabilidad de la biomasa seca total está influenciada por las variables antes mencionadas. Es evidente que las variables que mejor se correlacionaron con la biomasa forrajera seca, fueron: el número y longitud de los rebrotes y el Dap. Por lo que podrían considerarse como variables alométricas para predecir en campo la biomasa forrajera de esta especie. Las dos primeras variables demuestran que la capacidad de rebrotes es determinante en la acumulación de biomasa forrajera. Los valores de determinación encontrados para las variables diámetro de la base del rebrote y Dap sobrepasan significativamente a los reportados por López et al (2003), quienes encontraron para A. pennatula modelos no significativos y coeficientes de determinación menores a 0.3 , en un sitio usado como potrero forestal.
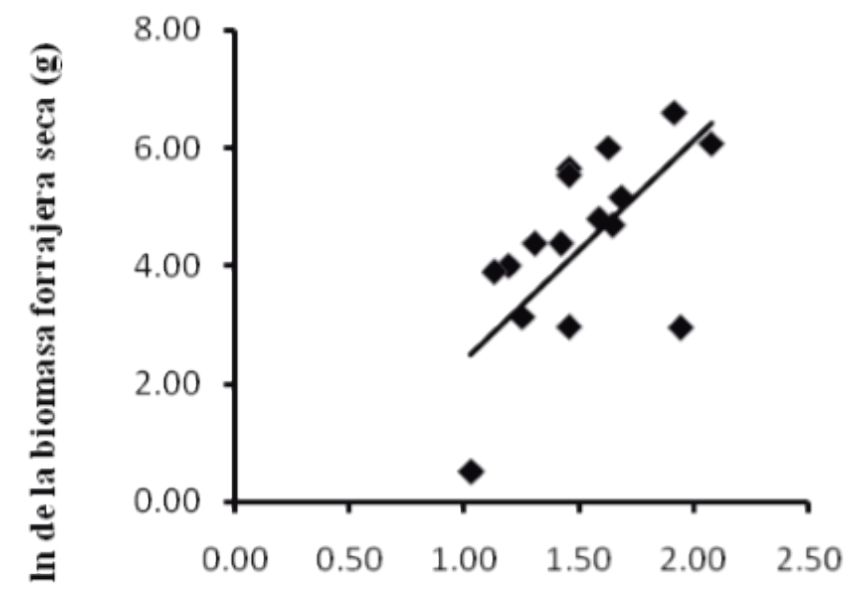

Diámetro de la base del rebrote $(\mathrm{mm})$

Figura 1. Biomasa forrajera seca según el diámetro de la base del rebrote en $A$. pennatula. $\mathrm{n}=17, \mathrm{p}=0.0036$.

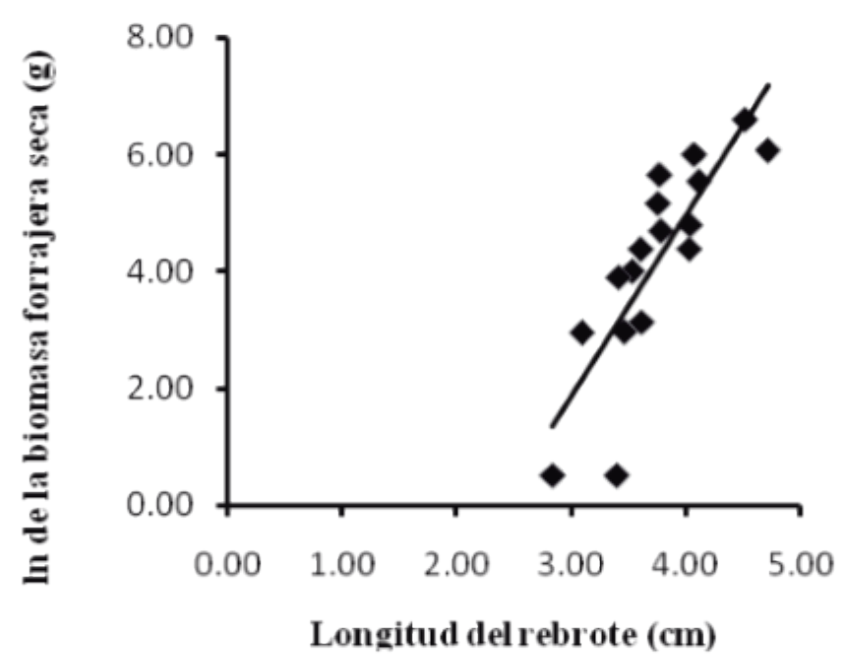

Figura 2. Biomasa forrajera seca según la longitud del rebrote en A. pennatula. n $=17, \mathrm{p}=0.0001$.

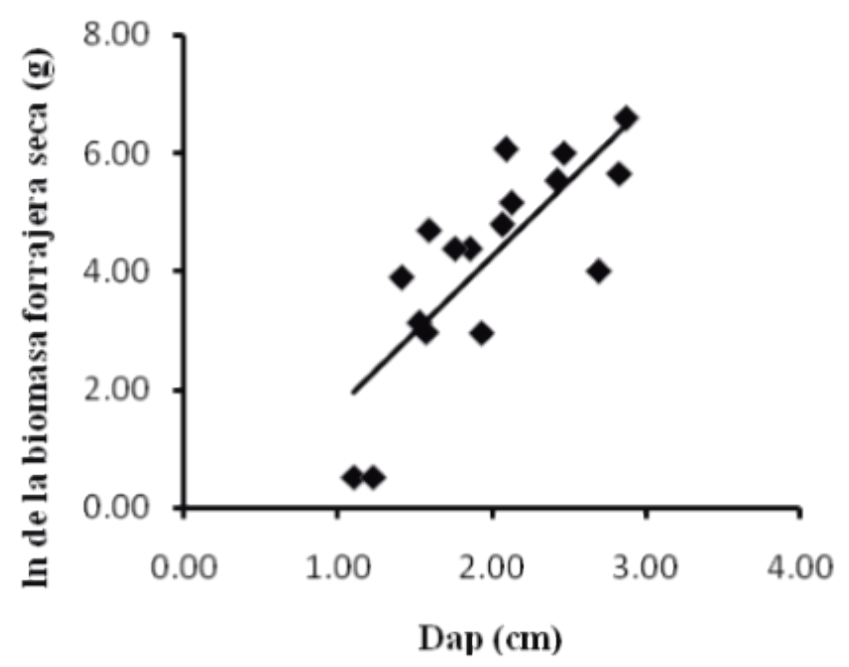

Figura 3. Biomasa forrajera seca según el Dap en A. pennatula. n $=17, \mathrm{p}=0.0002$. 


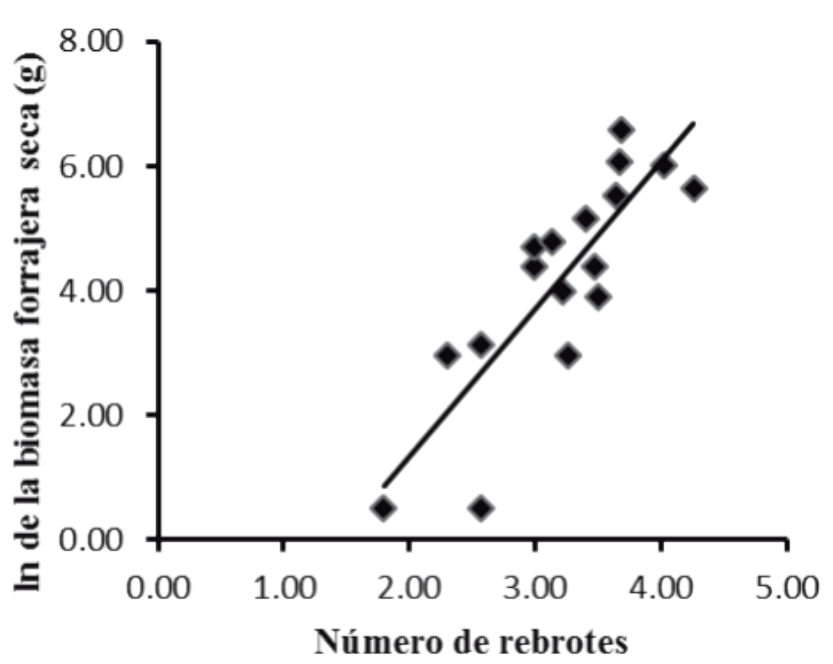

Figura 4. Biomasa forrajera seca según el número de rebrotes en A. pennatula. $\mathrm{n}=17, \mathrm{p}=0.0001$.

Los coeficientes de correlación de Pearson, demuestran un fuerte grado de asociación lineal positiva entre las variables que mejor determinaron la producción total por individuo de biomasa seca leñosa: diámetro de la base del rebrote y longitud del rebrote. Estas correlaciones resultaron estadísticamente significativas $(p<0.05)$ y se obtuvieron coeficientes de correlación cercanos a 1. Lo cual indica una relación lineal entre la producción de biomasa leñosa como variable dependiente del diámetro de la base y longitud del rebrote. No obstante la biomasa forrajera seca total por individuo no está asociada con la altura y el número de rebrotes, por presentar unos valores de correlación próximos a cero. A demás las correlaciones resultaron estadísticamente no significativa $(\mathrm{p}>0.05)$, (Tabla 3$)$.

Tabla 3: Correlación de la producción de biomasa leñosa seca total

\begin{tabular}{cccccc}
\hline $\begin{array}{c}\text { Correlación de } \\
\text { Pearson }\end{array}$ & $\begin{array}{c}\text { Diámetro de } \\
\text { la base del } \\
\text { rebrote } \mathbf{( c m )}\end{array}$ & $\begin{array}{c}\text { Longitud } \\
\text { del rebrote } \\
\mathbf{( c m})\end{array}$ & $\begin{array}{c}\text { Número } \\
\text { de rebotes }\end{array}$ & $\begin{array}{c}\text { Altura } \\
\mathbf{( m )}\end{array}$ & $\begin{array}{c}\text { Dap } \\
\mathbf{c m}\end{array}$ \\
\hline $\begin{array}{c}\text { Biomasa forrajera } \\
\text { seca total }(\mathrm{g})\end{array}$ & $0.617(* *)$ & $0.779(* *)$ & 0.265 & -0.337 & 0.524 \\
Sig. bilateral & 0.019 & 0.001 & 0.360 & 0.184 & 0.055 \\
$\mathrm{n}$ & 14 & 14 & 14 & 14 & 14 \\
\hline$*$ La correlación es significativa a nivel 0.05 (bilateral) & \\
$*$ * La correlación es significativa a nivel 0.01 (bilateral) &
\end{tabular}

Es evidente que la producción de biomasa forrajera y leña tuvieron un comportamiento lineal positivo para las variables alométricas evaluadas. Para la producción de leña, significa que a medida que estas aumentan estas variables, se obtiene mayor producción de leña (Figura 5, 6, 7 y 8). Es lógico que sea así, por que la producción primaria bruta (PPB) de la vegetación aumenta rápidamente hasta alcanzar un valor más o menos contante. Puesto que la energía radiante entra en el ecosistema por la superficie de las hojas y, una vez se alcanzado un cierto valor de índice foliar, las plantas ya no siguen acumulando hojas (Piñol y Martínez (2006).

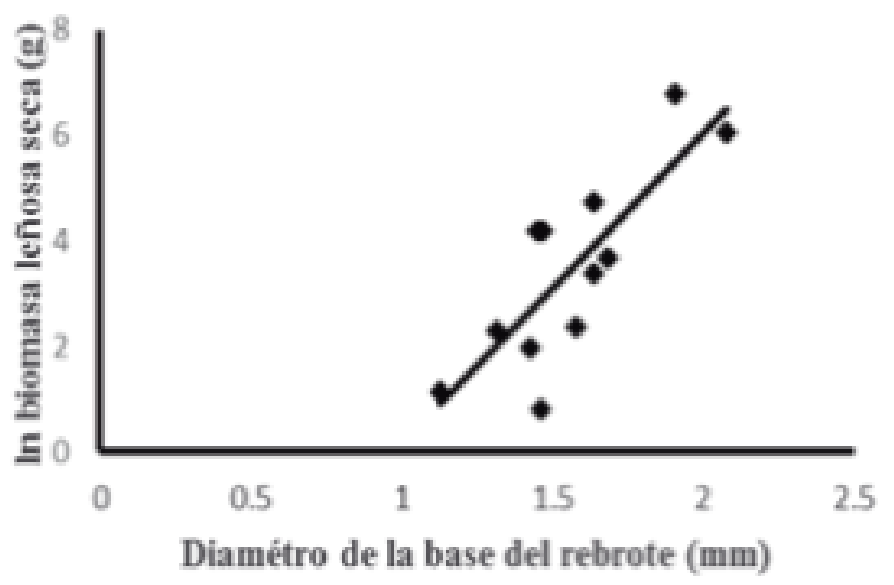

Figura 5. Biomasa leñosa seca según el diámetro de la base del rebrote en $A$. pennatula. $\mathrm{n}=12, \mathrm{p}=0.0013$. 


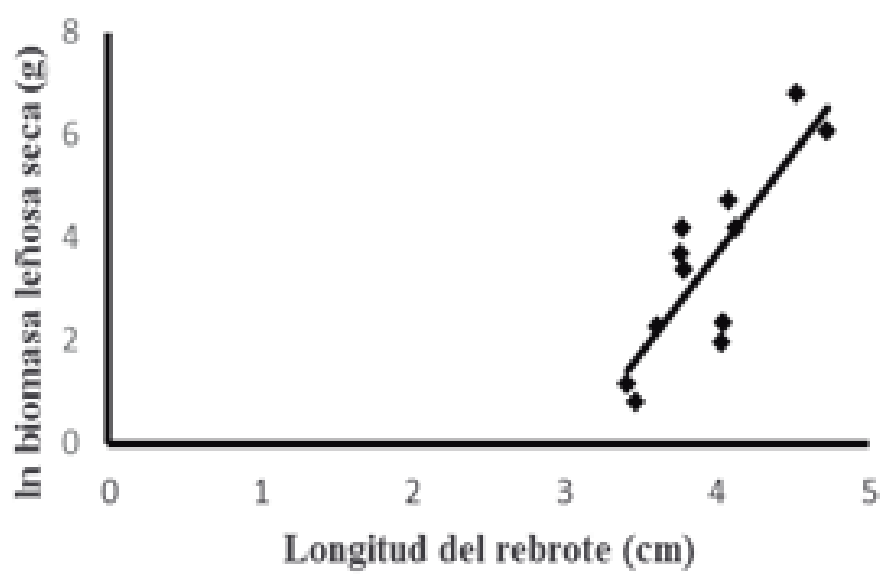

Figura 6. Biomasa leñosa seca según la longitud del rebrote en A. pennatula. $\mathrm{n}=12, \mathrm{p}=0.0007$.

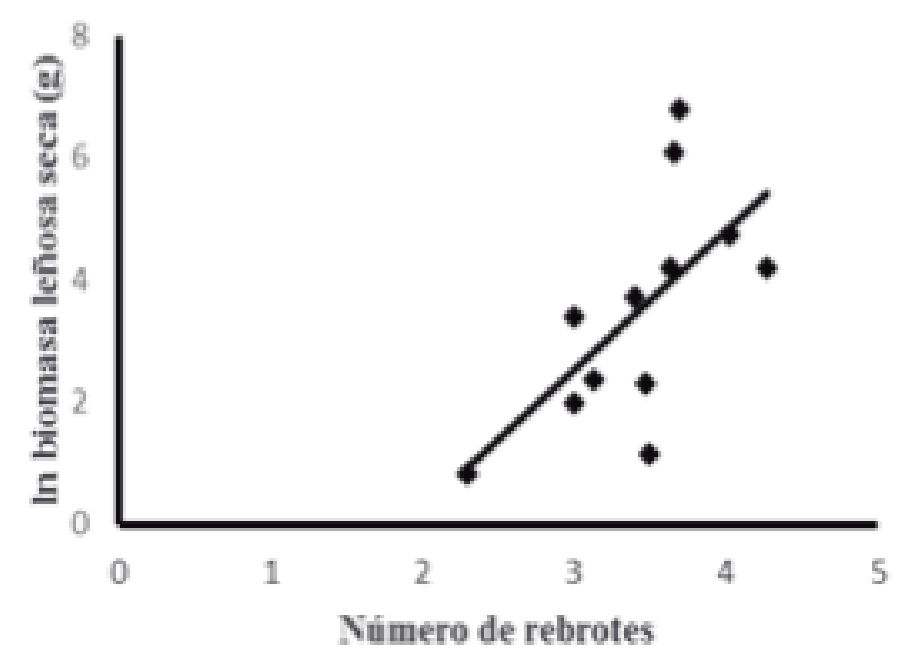

Figura 7. Biomasa leñosa seca según el número de rebrotes en A. pennatula. $\mathrm{n}=12, \mathrm{p}=0.0250$.

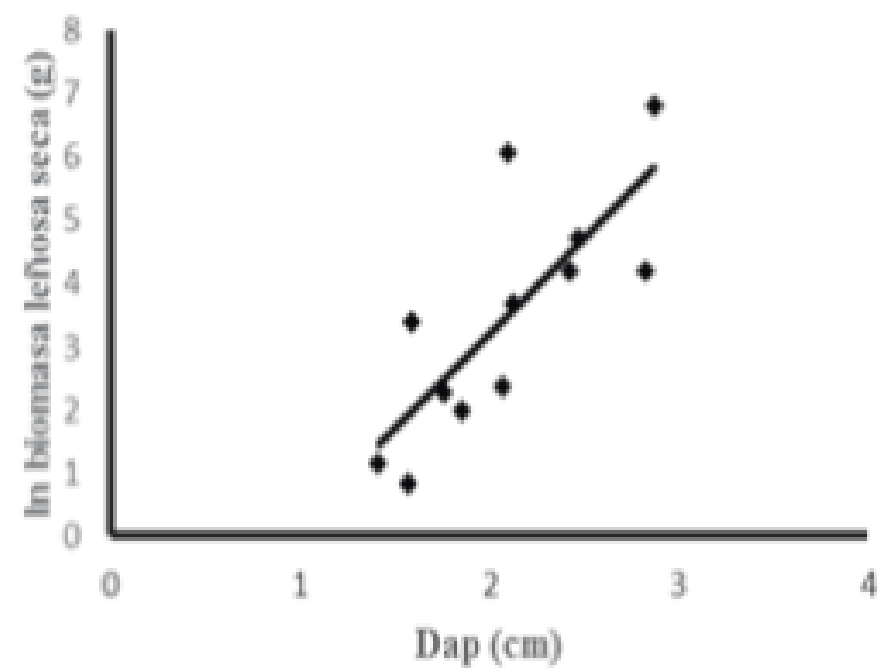

Figura 8. Biomasa leñosa seca según el número de rebrotes en A. pennatula. $\mathrm{n}=12, \mathrm{p}=0.0030$.

Los modelos alométricos generados, son aplicables únicamente a condiciones naturales de vegetación secundaria y características edafoclimáticas similares al sitio de estudio. Según los coeficientes de determinación (R2), las variables que mejor ajustaron la producción de material comestible fueron: longitud del rebrote y número de rebrotes. Los coeficientes indican que el forraje depende en un $70 \%$ y un $68 \%$, respectivamente, ambos modelos resultaron estadísticamente significativos. Mientras que la producción de leña se ajustó a la longitud del rebrote y diámetro de la base del rebrote (Tabla 5).

Modelos alométricos para la predicción de biomasa forrajera y leña. 
Tabla 5: Modelos de ecuaciones alométrias para la predicción de biomasa seca forrajera y leñosa en Carbón.

\begin{tabular}{|l|l|l|l|l|}
\hline $\begin{array}{l}\text { Variable } \\
\text { dependiente }\end{array}$ & $\begin{array}{l}\text { Variable } \\
\text { independiente }\end{array}$ & $\begin{array}{l}\text { Ecuaciones } \\
\text { alométricas }\end{array}$ & $\mathbf{R}^{2}$ & Significación \\
\hline \multirow{4}{*}{$\begin{array}{l}\text { Biomasa } \\
\text { forrajera } \\
\text { seca (g) }\end{array}$} & $\begin{array}{l}\text { Diámetro de la base } \\
\text { del rebrote }(\mathrm{cm})\end{array}$ & $\begin{array}{l}\mathrm{y}=3.7447 \mathrm{x}- \\
1.3735\end{array}$ & 0,4323 & 0.0036 \\
\cline { 2 - 5 } & $\begin{array}{l}\text { Longitud del rebrote } \\
(\mathrm{cm})\end{array}$ & $\begin{array}{l}\mathrm{y}=3.0727 \mathrm{x}- \\
7.3336\end{array}$ & 0,700 & 0.0001 \\
\cline { 2 - 5 } & Número de rebrotes & $\begin{array}{l}\mathrm{y}=2.3502 \mathrm{x}- \\
3.3439\end{array}$ & 0.684 & 0.0001 \\
\cline { 2 - 5 } & Altura (m) & $\begin{array}{l}\mathrm{y}=5.5403 \mathrm{x}- \\
6.6572\end{array}$ & 0,1633 & 0.1175 \\
\cline { 2 - 5 } & $\begin{array}{l}\text { Diámetro a la altura } \\
\text { de pecho (cm) }\end{array}$ & $\begin{array}{l}\mathrm{y}=2.5437 \mathrm{x}- \\
0.832\end{array}$ & 0.5718 & 0.0002 \\
\hline \multirow{5}{*}{$\begin{array}{l}\text { Biomasa } \\
\text { leñosa seca } \\
\text { (g) }\end{array}$} & $\begin{array}{l}\text { Diámetro de la base } \\
\text { del rebrote (cm) }\end{array}$ & $\begin{array}{l}\mathrm{y}=5.9112 \mathrm{x}- \\
5.7649\end{array}$ & 0.66 & 0.0013 \\
\cline { 2 - 5 } & $\begin{array}{l}\text { Longitud del rebrote } \\
\text { (cm) }\end{array}$ & $\begin{array}{l}\mathrm{y}=3.9168 \mathrm{x}- \\
11.954\end{array}$ & 0.7001 & 0.0007 \\
\cline { 2 - 5 } & Número de rebrotes & $\begin{array}{l}\mathrm{y}=2.2913 \mathrm{x}- \\
4.3639\end{array}$ & 0.4094 & 0.0250 \\
\cline { 2 - 5 } & Altura (m) & $\begin{array}{l}\mathrm{y}=195.47 \mathrm{x} 2 \\
-3160 \mathrm{x}+ \\
12666\end{array}$ & 0.3595 & 0.2384 \\
\cline { 2 - 5 } & $\begin{array}{l}\text { Diámetro a la altura } \\
\text { de pecho (cm) }\end{array}$ & $\begin{array}{l}\mathrm{y}=2.9831 \mathrm{x}- \\
2.7523\end{array}$ & 0.6023 & 0.0030 \\
\hline
\end{tabular}

Dónde: "y" = Biomasa forrajera y leñosa; a, b y c = parámetros a estimar $\mathrm{y}$ " $\mathrm{x}$ " = al valor que asuma cada variable independiente.

\section{CONCLUSIONES}

La mayor proporción de biomasa aérea, estuvo representa por la producción de forraje en relación a la leña.

Las variables regresoras longitud del rebrote, número de rebrotes y diámetro de la base del rebrote tienen la suficiente confiabilidad estadística para la estimación forraje y leña, en condiciones similares.

\section{REFERENCIAS BIBLIOGRÁFICAS}

Durr, P. (1992). Manual de árboles forrajeros de Nicaragua. MAG/COSUDE . 125 pp.

E, Pérez. (2006). Caracterización de sistemas silvopastoriles y su contribución socioeconómica a productores ganaderos. Tesis Mag. Sc. Copán,
Honduras. 138 p.

Etchevers, J; Vargas, J; Acosta, M; Velásquez, A. (2002). Estimación de la biomasa aérea mediante el uso de relaciones alométricas en seis especies arbóreas en Oaxaca, México. Agrociencia (Méx.) 36(6): $725-736$.

Gonzáles, M. (2008). Estimación de la biomasa aérea y la captura de carbono en regeneración natural de Pinus maximinoi, Pinus oocarpa y Quercus sp. Tesis Mag. Sc. Turrialba, Costa Rica. 97 p.

Guillén, A; Palacios, A; Espinoza, J. (2007). Ecuaciones de predicción para la producción de forraje de palo verde (Cercidium floridum) en baja California sur, México. Interciencia (Ven.) 32(10): 712 - 715.

Jiménez, C. (2010). Uso de ecuaciones alométricas para estimar biomasa y carbono en la parte aérea de Pinus hartwegii. Tesis Mag. Sc. Chapingo Texcoco, México. 65 p.

L. Simón, L. Lamela, D. Hernández. (2011). Los Sistemas Silvopastoriles en Cuba: Conferencia del 1er Seminario Internacional de Ganadería Agroecológica realizado en Villavicencio. (Col). Revista en Sistemas de Producción Agroecológica. 2(1): 22 pág.

M. Ibrahim, C. Villanueva, F. Casasolay J. Rojas. (2006). Sistemas silvopastoriles como una herramienta para el mejoramiento de la productividad y restauración de la integridad ecológica de paisajes ganaderos. Pastos y forrajes (Cub) 29(4): 383 - 489.

Nieto Villalobos, H. (2000). Contribución de Acacia pennatula a la productividad agroforestal sostenible de la reserva natural Miraflor Moropotente, Estelí, Nicaragua. Tesis Mag. Sc. Turrialba, Costa Rica. $69 \mathrm{p}$.

Peguero, G; Lanuza, O. R; Savé, R y Espelta, J. M. (2011). Allelopathic potential of the neotropical dry-forest tree Acacia pennatula Benth.: inhibition of seedling establishment exceeds facilitation under tree canopies. Plant Ecol.

Pérez, N y Linares, T. (2008). Sistemas agroforestales: una propuesta para la caracterización y evaluación de sistemas silvopastoriles. [En 
línea]. $1^{\circ}$ de Diciembre de Cali Colombia. www. agroforesteriaecologica.com.

Pérez, E. (2006). Caracterización de sistemas silvopastoriles y su contribución socioeconómica a productores ganaderos. Tesis Mag. Sc. Copán, Honduras. $138 \mathrm{p}$.

Piñol, J., \& Martínez Vilalta, J. (2006). Ecología con números. Una introducción a la ecología con problemas y ejercicios de simulación. Editorial Lynx, Bellaterra - Barcelona, España.

Silvina, M; Franco, J; Núñez, V; y Seghezzo, L. (2009). Estimación de densidad de biomasa aérea en ecosistemas naturales de la provincia de salta. ASADES (Arg.) 13: 37 - 45.

Stür, W.W.; Shelton, H.M. \& Gutteridge, R.C. (1994). Defoliation and management of forage tree legumes. In: Forage tree legumes in tropical agriculture. (Gutteridge, R.C. and Shelton, H.M., Eds.). CAB International. Wallingford, UK. p. 144. Salazar, R. (1989). Guía para la investigación silvicultural de especies de uso múltiple. Serie de investigación tecnica. Boletin tecnico 20. Centro de Agronomico de Investigación y Enseñanza (CATIE). Turrialba, Costa Rica. 